\title{
Compreendendo os Profissionais de Saúde da Familia como Potenciais Estudantes na Educação à Distância
}

\author{
Family Health Professionals as Potential \\ Distance Education Students
}

José Batista Cisne Tomaz Henk T. Van Der Molen

PALAVRAS-CHAVE

- Educação a Distância

- Educação Continuada

- Saúde da Família

KEYWORDS:

- Education, Distance

- Education, Continuing

- Family Health

Recebido em: 23/08/2010 Aprovado em: 10/12/2010

\begin{abstract}
RESUMO
Este artigo descreve os principais achados de um inquérito realizado no Estado do Ceará, Brasil, com o objetivo de avaliar a aceitabilidade de um curso baseado em Educação à Distância entre os profissionais que atuam no Programa Saúde da Família — uma nova estratégia para prover Atenção Primária à Saúde para a população brasileira. Foram enviados 255 questionários aos potenciais sujeitos, com uma taxa de resposta de 81,9\%. Os achados mostraram que, em geral, os respondentes têm percepções e atitudes positivas em relação à EAD e estão motivados em participar num curso baseado nessa estratégia. Propõe-se uma lista de recomendações para ajudar os planejadores do curso.
\end{abstract}

\section{ABSTRACT}

This paper reports the main findings of a cross-sectional survey in the State of Ceará, Brazil, aiming to assess the acceptability of a distance education (DE) course among health professionals working in the Family Health Program, a new strategy to provide primary care to the Brazilian population. A total of 255 questionnaires were sent to potential subjects, with a response rate of $81.9 \%$. Most respondents expressed positive perceptions and attitudes towards DE and were motivated to participate in a DE course. A list of recommendations was produced to help DE course planners. 


\section{INTRODUÇÃO}

O que você faria se fosse responsável pelo treinamento de um considerável número de pessoas (em torno de mil), que moram em diferentes lugares, algumas delas a centenas de quilômetros de sua instituição, num período de tempo relativamente curto? Educação à Distância (EAD) poderia ser a resposta. Entretanto, programas de EAD, em geral, não são tão fáceis de implantar e, usualmente, os custos iniciais tendem a ser muito altos $^{1}$ Infelizmente, alguns programas são implantados, grandes mudanças nas estratégias educacionais são feitas sem uma adequada avaliação de sua viabilidade, e muito dinheiro e esforço são desperdiçados. Portanto, três questões deveriam ser respondidas antes de se implantar um programa educacional baseado em $\mathrm{EAD}^{2}$ : primeiro, se essa estratégia seria aceitável para os alunos (ou potenciais alunos); segundo, se seria efetiva em ajudar os alunos no alcance dos objetivos educacionais; terceiro, mesmo se o programa educacional à distância for aceitável e efetivo, seria ele também eficiente - seria realístico e valeria a pena o gasto de tempo, energia e recursos financeiros por parte do usuário e de quem oferece o programa? $\mathrm{O}$ presente estudo é uma tentativa de investigar a primeira das três questões levantadas.

O objetivo deste estudo é descrever as características e percepções dos profissionais de Saúde da Família como potenciais alunos em um curso à distância no Estado do Ceará, Brasil. A principal questão a ser respondida é: a Educação à Distância (EAD) é uma estratégia educacional aceitável para treinar profissionais de Saúde da Família no Estado do Ceará sob a perspectiva dos potenciais alunos? Para responder esta questão, algumas perguntas mais específicas foram investigadas:

- Quem são os profissionais de Saúde da Família? Quais são suas principais características (idade, sexo, estado civil, profissão, histórico de formação educacional, etc.) e qual a relação dessas características com a EAD no Estado do Ceará?

- Quais são as principais percepções dos profissionais de Saúde da Família em termos de suas necessidades, preferências e contexto e qual a relação dessas percepções com a EAD no Estado do Ceará?

- Quais são as principais atitudes dos profissionais de Saúde da Família em relação à EAD como uma estratégia de educação permanente no Estado do Ceará?

- Que recomendações baseadas nos resultados podem ser propostas para ajudar os planejadores na elaboração do curso à distância, tornando-o mais aceitável aos alunos?

\section{MÉTODO}

Este estudo é um inquérito transversal, com abordagem quantitativa. O desenho é parcialmente adaptado do trabalho desenvolvido por Terry Evans ${ }^{3}$, da Faculdade de Educação da Universidade de Deakin, Austrália. Em seu trabalho, ele investigou em detalhe uma variedade de temas relacionados, desde o histórico educacional e social dos estudantes a outros importantes aspectos das vidas dos alunos e suas inter-relações com seus contextos.

Entretanto, o presente estudo se diferencia do trabalho desenvolvido por Evans em um importante aspecto: o estudo de Evans foi realizado entre estudantes que já tinham tido experiência em cursos baseados em EAD. Em nosso estudo, a maioria dos alunos não teve experiência prévia em EAD.

A população deste estudo foi constituída por médicos e enfermeiros de família do Programa Saúde da Família (PSF) do Estado do Ceará em 2006 e 20074-7, excluindo aqueles que já haviam feito ou estavam fazendo o Curso de Especialização em Saúde da Família (Cesf) - um curso presencial, baseado em problemas (Problem-Based Learning - PBL) ${ }^{8,9}$, oferecido pela Escola de Saúde Pública do Ceará (ESP-Ce). Assim, excluindo tais profissionais, o número final foi de 881 . Desse número foram selecionados aleatoriamente 115 médicos de família e 140 enfermeiros de família.

Um questionário autoaplicável foi desenvolvido especificamente para este estudo. Ele foi organizado em cinco blocos: dados pessoais; histórico social e de formação educacional dos potenciais estudantes; necessidades, percepções e contexto dos potenciais estudantes; desenho do curso; questões gerais. A maioria das questões foi fechada e elaborada em diferentes formatos. Nas questões utilizadas para medir atitudes e percepções, uma escala de cinco pontos (escala de Likert) foi usa$\mathrm{da}^{10}$. Um pré-teste foi realizado com quatro profissionais de Saúde da Família - dois médicos e duas enfermeiras - que não foram incluídos no estudo.

Foram enviados 255 questionários para todos os médicos de família $(\mathrm{n}=115)$ e todos os enfermeiros de família $(\mathrm{n}=$ 140) de 31 municípios selecionados aleatoriamente, excluindo os profissionais mencionados anteriormente. Todos os dados foram analisados por meio de um software estatístico - SPSS, versão 6.1.4. Foram utilizados principalmente procedimentos estatísticos descritivos, tais como frequências, incluindo média e desvio padrão, e procedimentos estatísticos inferenciais, como a tabulação cruzada, incluindo o nível de significância ( $p$ value).

\section{RESULTADOS}

A taxa de resposta dos participantes foi de 81,9\% (209 de 255). As taxas de respostas por categoria profissional foram: 70,4\% 
(81 de 115) entre os médicos de família e 91,4\% (128 de 140) entre os enfermeiros de família. Os respondentes representaram 23,7\% do total da população do estudo (209 de 881 ).

Foi observado que uma importante maioria dos respondentes era do sexo feminino $-70,3 \%(n=147)$. Em relação à categoria profissional, a percentagem de enfermeiros de família $-61,2 \%(n=128)$ - foi significativamente maior que a de médicos de família $-38,8 \%(\mathrm{n}=81)$. A maioria dos respondentes era solteira $-45,7 \%(n=91)$ ou casada $-43,2 \%$ ( $n$ = 86). A distribuição da idade dos respondentes variou de 21 a 70 anos. A maioria estava entre 25 e 35 anos, e a média de idade foi de 32,2 anos.

A Tabela 1 apresenta as respostas dos participantes relacionadas ao histórico da formação educacional numa escala de cinco pontos $(1=$ discordo fortemente e 5 = concordo fortemente).

\section{TABELA 1}

Respostas dos participantes relacionadas ao histórico de formação educacional

\begin{tabular}{lcc}
\hline \multicolumn{1}{c}{ Item } & $\mathbf{M}$ & Sd \\
\hline $\begin{array}{l}\text { Eu tive uma experiência prévia positiva como } \\
\text { estudante }\end{array}$ & 4,17 & 0,84 \\
\hline $\begin{array}{l}\text { Minhas experiências prévias foram marcadas por } \\
\text { medo e falhas }\end{array}$ & 1,55 & 1,00 \\
\hline $\begin{array}{l}\text { Eu tenho estudado em escolas de má qualidade } \\
\text { Eu gosto muito de aprendizagem independente }\end{array}$ & 1,54 & 1,03 \\
\hline
\end{tabular}

Um importante achado neste tópico foi que quase metade dos participantes - 47,4\% ( $\mathrm{n}=98)$ - afirmou que não gostava de aprendizagem independente. Só 18,9\% $(n=39)$ responderam positivamente. Além disso, a grande maioria - 90,7\% (n = 186) - não teve nenhuma experiência em EAD.

Em relação às necessidades, preferências, contexto, percepções e atitudes dos potencias estudantes, alguns resultados relevantes foram encontrados. No que se refere ao acesso aos recursos de aprendizagem, os achados mostraram que uma boa proporção dos respondentes — 59,3\% $(n=124)$ — tinha fácil acesso a computador, considerando ao menos uma versão PC Pentium I ou similar ou maior. Entretanto, uma considerável porção - 40,7\% ( $\mathrm{n}=85)$ - não tinha. A maioria - 71,3\% ( $n=149)$ — não tinha acesso fácil à internet, e 64,4\% ( $n=134)$ tinham fácil acesso à biblioteca ou a centros de estudo.

Em relação a algumas habilidades existentes, foi observado que quase a metade dos respondentes - 45,9\% ( $\mathrm{n}=$ 95) - afirmou que não era capaz de ler em inglês. Somente $29 \%(n=70)$ responderam positivamente. Em relação ao uso do software Word e ao uso do Excel, 47,1\% (n = 96) e 58,7\% ( $n=119)$ responderam que não eram capazes de utilizá-los, respectivamente. Por fim, os achados relacionados ao uso da internet mostraram que a grande maioria $-70,1 \%(n=143)$ — não era capaz de utilizá-la. Somente $18,1 \%(n=37)$ disseram que sim.

Um dos resultados interessantes relacionado ao tópico poder e controle foi que a grande maioria dos respondentes $90,4 \%$ (188) - concordou em que na EAD o estudante tem ao menos o poder de estudar num local e a qualquer tempo que ele possa escolher. Ademais, uma importante maioria - 83,5\% ( $n=172)$ - afirmou que gostaria de participar do planejamento de um provável curso baseado em EAD.

Em relação ao tópico trabalho, treinamento e educação, mais da metade dos respondentes - 56\% ( $\mathrm{n}=117)$ - disse que, em média, trabalhava 40 horas por semana. Em torno de $29 \%$ (n = 61) trabalhavam mais que 40 horas e menos de 60 horas, $\mathrm{e}$ somente $7,7 \%(n=16) 60$ horas ou mais, $M=2,37, S D=0,73$. Cruzando as variáveis profissão e horas trabalhadas, encontramos que um pouco mais da metade dos médicos de família - 51,9\% ( $\mathrm{n}=42)$ - disse que trabalhava mais que 40 horas por semana. Entre os enfermeiros, somente $27,3 \%(n=35)$ disseram que também trabalhavam mais que 40 horas semanais, $\mathrm{p}=0$.

Algumas respostas dos participantes relacionadas com trabalho, treinamento e educação são apresentadas na Tabela 2. Analisando os achados, destacamos três mais relevantes: podemos notar que $73,6 \%(n=153)$ disseram que, em geral, eram capazes de conciliar trabalho e estudo, considerando em média 20 horas de estudo por semana. Ademais, praticamente todos eles - 98,6\% ( $n=206)$ - afirmaram que havia uma importante relação entre o seu trabalho e o estudo.

Outro achado mostrou que uma grande maioria - 95,2\% ( $n=199)$ - concordou em que a combinação de EAD com atividades educacionais em seu local de trabalho poderia ser uma importante alternativa para treinar os profissionais do PSF.

Os resultados relacionados ao desenho do curso foram muito relevantes. Os achados mostraram que a grande maioria $-89,5 \%(n=187)$ - disse que gostaria de participar do curso baseado em EAD.

Os resultados relacionados às razões pelas quais eles gostariam de participar são mostrados na Figura 1. Entre as sete opções propostas no questionário, as três mais mencionadas foram: "Para minha melhoria em termos intelectuais", marcada por 87,6\% (n = 163); "Para obter qualificação para meu atual emprego", escolhida por 75,3\% ( $=140)$; e "Por prazer", selecionada por $66,1 \%(n=123)$. 
TABELA 2

Respostas dos participantes relacionadas com trabalho, treinamento e educação

\begin{tabular}{lcc}
\hline \multicolumn{1}{c}{ Item } & M & Sd \\
\hline $\begin{array}{l}\text { Em geral, eu sou capaz de conciliar trabalho e } \\
\text { estudo (considere uma média de 20 horas de estudo }\end{array}$ & 4,05 & 1,46 \\
\end{tabular}

semanalmente)

Em minha experiência, meu empregador tem

facilitado minha participação em cursos residenciais fora do município

Se os cursos mencionados no item anterior fossem baseados em EAD, meu empregador facilitaria mais facilmente minha participação

Eu sou capaz de conciliar atividades em grupo, tais como estudo em equipe e grupos tutoriais, com meu trabalho caso tais atividades sejam usadas em cursos baseados em EAD

Um misto entre EAD e atividades educacionais no local de trabalho poderia ser uma importante alternativa para treinar profissionais de Saúde da Família

Meu empregador me dá algum tempo para estudar durante meu horário de trabalho

FIGURA 1

Razões pelas quais os profissionais de Saúde da Família gostariam de participar do curso

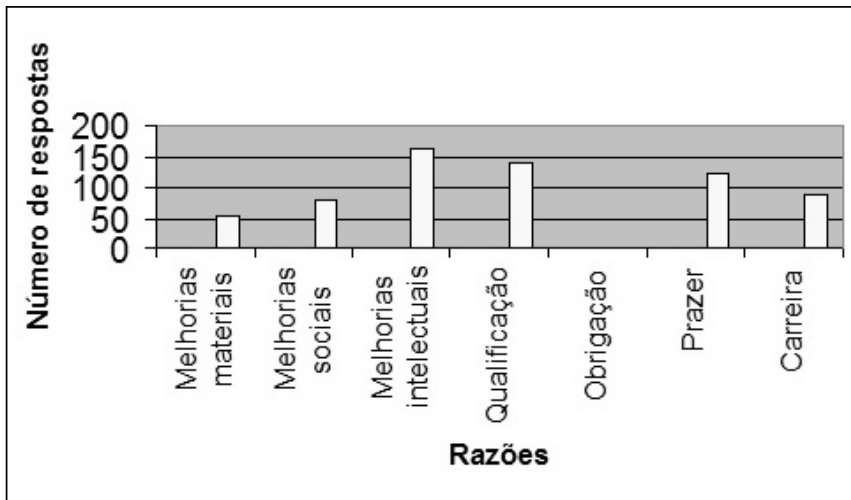

Os resultados a respeito da disponibilidade dos respondentes para pagar por um curso à distância mostraram que $56 \%(n=108)$ estavam dispostos a pagar pelo curso proposto. Parte deles $-22,8 \%(n=44)-$ respondeu que não. Uma significativa percentagem $-21,2 \%(n=41)-$ disse que "não sabia". Dentre os que estavam dispostos a pagar, a grande maioria - 92\% ( $n=115)$ - disse que em torno de $\mathrm{R} \$ 200,00$ (duzentos reais) por mês seria uma quantia razoável. Ademais, $71,5 \%$ $(\mathrm{n}=103)$ disseram que estavam dispostos a pagar por material educacional, como livros e apostilas, e $51 \%(n=73)$ estavam dispostos a pagar por custos de comunicação, como telefone, correio e internet.

A seguir, os respondentes escolheram, de uma lista de seis, as mídias que gostariam que fossem utilizadas no curso (Figura 2). As três mídias mais mencionadas foram: "material impresso", selecionada pela grande maioria $-94,3 \%$ (n = 197); "vídeo", escolhida por $88,5 \%$ (n = 185); e "computador", marcada por $59,8 \%(n=125)$. "Fita de áudio" foi marcada por somente $34 \%(n=71)$. Numa questão aberta, só $1,5 \%(n=3)$ preferiria outras mídias além das citadas no questionário.

FigURA 2

Preferências dos respondentes em relação às mídias

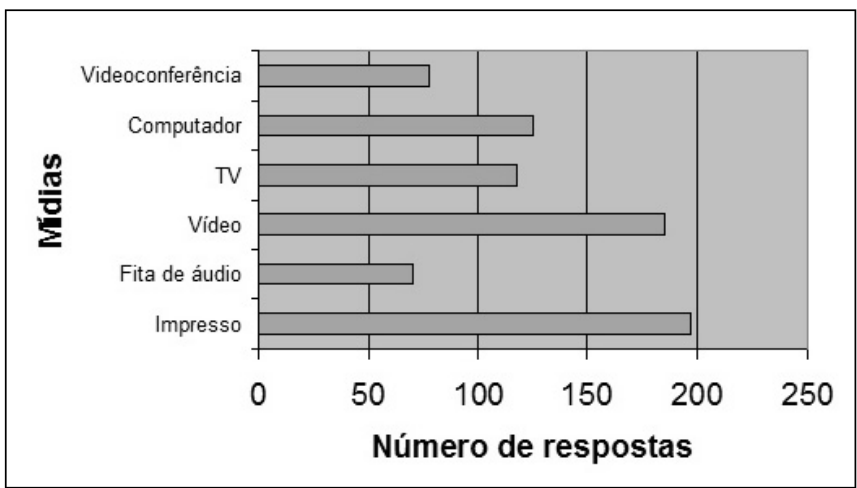

Perguntou-se também, usando a mesma lista, a quais mídias eles considerariam que tinham acesso mais fácil. Os resultados são mostrados na Figura 3. As três mais citadas foram: "vídeo", marcada pela grande maioria - 96,2\% ( $\mathrm{n}=200)$; "material impresso", também escolhida pela grande maioria - 93,3\% (n = 194); e "TV", selecionada por 75,5\% ( $=157)$. "Fita de áudio" foi escolhida por 41,3\% ( $n=86)$. "Computador" foi marcada por 49,5\% (n = 103). "Videoconferência" foi selecionada por somente $14,4 \%(n=30)$.

Três aspectos adicionais também foram investigados em relação à estrutura do curso e que são fundamentais na EAD: a flexibilidade da data-limite da entrega das avaliações, o uso de sessões presenciais e o treinamento de habilidades. Pode-se observar que $68,9 \%(n=144)$ concordaram em que a data das avaliações poderia ser flexível para possibilitar aos alunos a oportunidade de estudar em sua própria velocidade, de acordo com suas capacidades e circunstâncias. Ademais, uma proporção substancial dos respondentes - 80,8\% (n = 168) - concordou em que sessões presenciais, como estudos em grupo e grupos tutoriais, deveriam ser incluídas no curso para estimular o contato entre os participantes. Finalmente, 58,6\% ( $n=119$ ) concordaram em que o treinamento de habilidades deveria ocorrer, preferencialmente, em sessões presenciais. 
Os resultados relacionados à motivação e interesse foram bem relevantes. Observou-se que a maioria $-77,7 \%(n=160)$ - afirmou que se sentia motivada ou muito motivada para participar do curso proposto. Quando perguntados sobre o grau de interesse no tema "Saúde da Família", quase todos os respondentes - 98,5\% $(n=190)$ - disseram que sim.

Figura 3

Respostas dos participantes em relação ao acesso às mídias

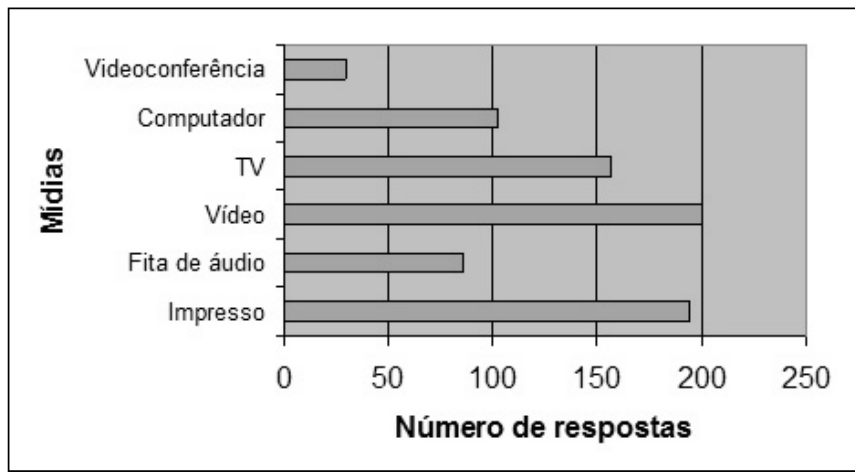

Um último achado bem interessante foi que mais da metade dos respondentes $-67,9 \%(n=125)-$, se pudesse escolher, preferiria participar do curso proposto utilizando a estratégia de $\mathrm{EAD}$, ao invés da abordagem tradicional (presencial) $-32,1 \%(n=59)$.

\section{DISCUSSÃO}

As bases teóricas do estudo, adaptadas de Evans ${ }^{3}$, se mostraram efetivas para o nosso propósito. Outros aspectos adicionados, baseados em nossa própria experiência e necessidades, também pareceram ser adequados. Em termos gerais, o questionário pareceu ser efetivo e mediu o que o estudo se propôs medir. Apesar de seu tamanho (70 itens), as questões foram fáceis de responder. O pré-teste foi de muita importância para fazer os ajustes.

Em relação aos resultados, um dos principais achados deste estudo está relacionado ao desenho do curso, particularmente no que se refere ao desejo de participação do curso baseado em EAD e às suas razões. De fato, tais achados são de muita importância para responder se a EAD é uma estratégia aceitável a ser usada na educação permanente dos profissionais de Saúde da Família. Os resultados mostraram claramente que a grande maioria gostaria de participar do curso em Saúde da Família usando a estratégia de EAD.

Analisando as razões para participar do curso, observa-se que duas das três opções mais selecionadas - "para minha melhoria em termos intelectuais" e "por prazer" — indicam que tais respondentes têm motivação acadêmica/intrínseca e motivação pessoal/intrínseca, respectivamente. A outra opção - "para obter qualificação para meu atual emprego" — demonstra motivação vocacional/extrínseca. Motivação ou orientação para a aprendizagem tem sido definida como todas as atitudes, objetivos e propósitos que expressam uma relação do estudante com um curso e com a instituição de ensino ${ }^{11}$. As motivações intrínsecas e extrínsecas dos estudantes para participar de um curso têm impacto crucial no grau de envolvimento e dedicação às atividades do curso ${ }^{12}$.

Alguns autores (Sagar e Strang, 1985; Strang, 1987, citados em Rowntree ${ }^{13}$, 1992, p. 132) sugerem que estudantes com motivação intrínseca tendem a alcançar melhores notas do que aqueles que têm interesses extrínsecos. Esses mesmos autores também propõem que alunos que têm várias razões para estudar apresentam melhor desempenho do que os que têm somente uma. Esses estudos também sugerem que a motivação intrínseca estimula os estudantes a obter um hábito de aprendizado "para toda a vida" (lifelong learning), o que deve ser o objetivo principal de qualquer sistema educacional.

Outro interessante resultado relacionado ao desenho do curso é a disponibilidade para pagar pelo curso, pelo material didático e pelos custos de comunicação. Este fato pode ser um sinal de motivação dos respondentes para participar do curso. Planejadores e gerentes podem levar em conta esta informação ao elaborarem o orçamento do curso.

Em relação às mídias a serem usadas no curso, as quatro mais selecionadas foram: material impresso, vídeo, computador e TV. Esta informação pode dar importantes sugestões aos planejadores do curso ao selecioná-las. De acordo com a literatura ${ }^{13,3,14}$, o material impresso ainda é uma importante mídia escolhida pelos estudantes.

Outro achado que pode ajudar os planejadores no desenho do curso é que, em geral, os participantes do estudo demonstraram ter uma atitude positiva em relação a três aspectos investigados relacionados com a estrutura do curso e que são fundamentais na EAD: a flexibilidade da data da entrega das avaliações, o uso de sessões presenciais, como estudos em grupos e grupos tutoriais no curso, e a realização do treinamento de habilidades, preferencialmente, em sessões presenciais.

Os resultados relacionados ao histórico social e educacional dos potenciais alunos também nos permitem fazer alguns comentários. Primeiro, a maioria dos profissionais de Saúde da Família, em geral, teve uma experiência prévia positiva como estudantes. Este fato é muito importante, principalmente para a EAD, porque tais estudantes tendem a ser mais bem-sucedidos em futuros cursos ${ }^{3}$. Por outro lado, a maioria dos profissionais participantes do estudo não teve experiência em 
aprendizagem independente. Este achado não é tão positivo para a EAD, já que aprendizagem independente é uma importante característica dessa estratégia. Talvez eles tenham que se adaptar ou serem estimulados a utilizar a aprendizagem independente caso participem de um curso baseado em EAD.

Outro aspecto que merece um comentário é que a maioria dos profissionais de Saúde da Família não teve experiência prévia em $\mathrm{EAD}$, mas aqueles que tiveram tal experiência gostaram muito e tiveram boas relações com os tutores. De fato, a EAD é totalmente diferente da educação tradicional. Os estudantes que participam de um curso baseado em EAD pela primeira vez devem ser alertados para manter certa disciplina em termos de tempo, local de estudo e conciliação entre família, trabalho e lazer. O contato com os colegas (por telefone, internet ou mesmo pessoalmente) é também uma importante estratégia para manter um bom desempenho em cursos baseados em EAD.

Quanto a necessidades, preferências, contexto, percepções e atitudes dos potenciais estudantes, em particular o acesso aos recursos de aprendizagem, os resultados mais importantes mostram que uma pequena maioria dos profissionais de Saúde da Família tem acesso fácil a computador e a bibliotecas ou centros de estudo. Este é um ponto fundamental na EAD e indica a possibilidade de usar tais recursos de aprendizagem no curso. Por outro lado, eles têm pouco acesso à internet $28,7 \%(n=60)$. A internet é usada na EAD de diferentes maneiras - comunicação entre colegas, estudantes e tutores, e como uma infinita fonte de informação. Talvez algum programa governamental possa estimular o aumento desse percentual.

Em termos de habilidades existentes, os resultados mostram que quase metade dos profissionais de Saúde da Família não se considera capaz de ler em inglês. De fato, muito material impresso e importantes informações na internet estão na língua inglesa. Assim, é fundamental que os profissionais de Saúde da Família tenham uma razoável capacidade para ler em inglês. Esta pode ser uma importante limitação na EAD para estes profissionais. Em relação às outras habilidades existentes investigadas - capacidade para utilizar os softwares Word, Excel e internet - , os achados mostram que, em geral, eles têm limitações, principalmente no uso da internet. É interessante observar que somente $35,8 \%$ dos respondentes disseram que são capazes de utilizar o Word e 18,1\% que são capazes de usar a internet, embora 59,3\% tenham dito que tinham acesso a computador e 28,7\% à internet. Algumas estratégias poderiam ser propostas para tentar superar essas dificuldades, tais como a promoção de tradução para o inglês de parte do material impresso, a elaboração de textos em português ou a estimulação desses profissionais para participar de cursos de inglês e de informática.
Os resultados no tópico poder e controle também mostram que os respondentes têm uma atitude favorável em relação à EAD. De fato, eles acham que a EAD provê aos alunos ao menos o poder de estudar num local e a qualquer tempo que possam escolher, e que a distância entre os estudantes e as instituições não contribui para a falta de poder deles. Esses resultados mostram, até certo ponto, que o problema de poder e controle existe no ambiente educacional e que a EAD pode minimizá-lo, pelo menos em alguns aspectos. O conceito de poder e controle significa que boa parte das decisões e atividades curriculares está centrada na instituição de ensino e no professor, tendo o aluno pouco controle sobre sua aprendizagem. Esta reflexão nos remete ao conceito de modelo educacional centrado no aluno, no qual o currículo tem o foco nas necessidades do aluno, e a abordagem metodológica estimula a participação ativa do aluno no processo de ensino-aprendizagem, como na Aprendizagem Baseada em Problemas (Problem-Based Learning - PBL) ${ }^{8}$. Assim, apesar de a EAD, por si só, contribuir para minimizar a questão do poder e controle no ambiente educacional, é importante que a proposta pedagógica utilize abordagens centradas no aluno, de modo que ele tenha maior controle e poder em seu processo de aprendizagem.

No estudo de Evans ${ }^{3}$, uma variedade de estudantes estava também consciente deste tipo de problema e de sua falta de poder no ambiente educacional. Os resultados também mostram que os respondentes desejam compartilhar o poder e o controle com os professores e com a instituição, por meio da manifestação de seu desejo de participar no planejamento do curso, por exemplo.

Os resultados relacionados a trabalho, treinamento e educação são também, de certa maneira, favoráveis à implantação de um curso à distância em nosso contexto. A maioria de nossos potenciais alunos, em particular os enfermeiros de família, disse trabalhar, em média, 40 horas por semana ou menos e, predominantemente, tem somente um emprego público. De acordo com Andrade ${ }^{15}$, 85,2\% dos médicos de família e 92,1\% dos enfermeiros de família no Ceará dedicavam, em média, 40 horas por semana ao PSF na época do estudo, o que é congruente com nossos achados. Por outro lado, os resultados também mostram que os profissionais de Saúde da Família parecem dispostos a conciliar trabalho e ao menos 20 horas de estudo semanais, e acham que o estudo tem influenciado consideravelmente seu trabalho. A conclusão positiva é que, até certo ponto, os respondentes parecem ter tempo suficiente para se matricular num curso baseado em EAD.

Finalmente, outro valioso achado em nosso estudo, talvez um dos mais importantes, é que os respondentes demonstraram estar muito motivados para participar do curso baseado em EAD e muito interessados no tópico "Saúde da Família". 


\section{RECOMENDAÇÕES}

Um dos objetivos do presente estudo foi identificar e propor recomendações para ajudar os planejadores no desenho de curso baseado em EAD, tornando-o mais aceitável para os potenciais alunos. Algumas recomendações já foram propostas na discussão. Nesta seção, iremos condensá-las para facilitar sua leitura e uso, e acrescentar algumas outras. Focaremos as recomendações relacionadas com os resultados.

(a) Ao planejar o curso baseado em EAD, deve-se considerar o perfil predominante dos potenciais alunos: sexo feminino, enfermeiro, solteiro e jovem. Naturalmente, os outros não devem ser esquecidos.

(b) Como a grande maioria dos potenciais estudantes não tem experiência anterior com EAD, eles devem receber algumas recomendações no começo ou no decorrer do curso, (por exemplo, como ser um bom estudante em cursos baseados em $\mathrm{EAD}^{3}$ ).

(c) $\mathrm{O}$ contato entre colegas e entre alunos e professores (por telefone, internet ou mesmo pessoalmente) deve ser estimulado durante o curso.

(d) Os gerentes e planejadores do curso devem propor programas ao governo para estimular e subsidiar a aquisição de computadores e o acesso à internet aos profissionais de Saúde da Família como parte de um projeto de educação permanente. Bibliotecas e centros de estudo poderiam ser incluídos no projeto.

(e) A tradução de parte do material impresso do inglês para o português poderia ser promovida, textos em português poderiam ser elaborados, e os profissionais de Saúde da Família poderiam ser estimulados a participar de cursos de inglês e de informática, talvez usando a própria estratégia de EAD.

(f) Quando viável, representantes dos estudantes devem ser incluídos no planejamento do curso baseado em EAD. A distância entre a instituição e o estudante pode contribuir para o seu sentimento de falta de poder.

(g) Os profissionais de Saúde da Família sempre têm muitas atividades a realizar. Assim, não se deve sobrecarregá-los com muitas tarefas, em particular os médicos que parecem ter muito trabalho extra.

(h) Ao selecionar as mídias para o curso, devem-se levar em consideração as preferências dos potenciais estudantes e o acesso às mídias. Assim, material impresso, vídeo, computador e TV podem ser as melhores escolhas em nosso caso.

(i) Em relação à estrutura do curso, deve-se estimular a possibilidade de dar alguma flexibilidade à data de entrega das avaliações, usar sessões presenciais, como estudos em grupo e grupos tutoriais, e planejar o treinamento de habilidades em sessões presenciais.

\section{CONCLUSÕES}

Este estudo investigou um dos aspectos mais importantes para a implantação de um curso baseado em EAD: a aceitabilidade do curso por parte dos potenciais alunos, no caso, profissionais do Programa Saúde da Família. As bases teóricas do estudo, adaptadas de Evans $^{3}$, se mostraram efetivas para o nosso propósito. Importantes informações foram coletadas, incluindo características, percepções, opiniões, preferências e atitudes dos potenciais alunos.

Os principais resultados mostraram que a maioria dos participantes do estudo não teve experiência prévia com a EAD e que, apesar de ter acesso a computador, boa parte deles não tem acesso à internet, o que pode ser uma limitação na EAD. No entanto, vários outros achados mostraram que a EAD pode ser uma estratégia adequada à educação permanente dos profissionais de Saúde da Família. O desejo de participar no planejamento do curso, a motivação para participar de um curso baseado em EAD e a disponibilidade para pagar por ele são exemplos desses achados. Material impresso, vídeo e computador foram os meios preferidos pelos participantes do estudo. Outra conclusão positiva é que, até certo ponto, os potenciais alunos parecem estar dispostos a dedicar tempo suficiente para participar do curso baseado em EAD. Todas essas informações devem ser levadas em conta pelos planejadores ao desenharem o currículo e o material didático do curso.

Em suma, de maneira geral, duas conclusões principais podem ser tiradas deste estudo: (a) os profissionais de Saúde da Família demonstraram ter percepções e atitudes positivas em relação à EAD e (b) uma série de informações relevantes deve ser considerada pelos planejadores do curso baseado em EAD antes de implementá-lo. Futuros estudos devem ser realizados, incluindo opiniões e percepções de outros atores envolvidos, como gestores de saúde e educadores.

\section{REFERÊNCIAS}

1. Rumble G. The costs and economics of open and distance learning. London: Kogan Page; 1997.

2. Engel CE, Browne E, Nyarango P, Akor S, Khwaja A, Karim AA, et al. Problem-based learning in distance education: a first exploration in continuing medical education. Med Educ. 1992;26:389-401.

3. Evans T. Understanding learners in open and distance education. London: Kogan Page; 1994.

4. Ministério da Saúde. Saúde Dentro de Casa: Programa Saúde da Família. Brasília: MS;1994. 
5. Secretaria de Saúde do Estado do Ceará. Saúde da Família: um novo modelo de atenção. Fortaleza: Escola de Saúde Pública do Ceará; 1994.

6. World Health Organization. Making medical practice and education more relevant to people's needs: the contribution of the family doctor. Genebra: Anais da WHO-WONCA Conference;1994.

7. Ministério da Saúde. Programa de Saúde da Família: Projeto do Pólo de Capacitação, Formação e Educação Permanente de Pessoal Estratégico para a Saúde da Família no Ceará. Brasília: MS;1997.

8. Schmidt HG. Problem-Based Learning (PBL): Rationale and Description. Med Educ. 1983;17: 11-6.

9. Magzoub M. A comprehensive evaluation of the Diploma Course in Family Health and Diploma Course in Local Health System Management of School of Public Health in State of Ceará. Fortaleza: Escola de Saúde Pública do Ceará; 1998.

10. Crowl TK. Fundamentals of Educational Research. London: Brown \& Benchmark Publishers; 1996.

11. Taylor E, Morgan AR, Gibbs G. Teaching at a distance; 1981.

12. Morgan A. Improving your students' learning: reflections on experiences of study. London: Kogan Page; 1993.
13. Rowntree D. Exploring open and distance learning. London: Kogan Page; 1992.

14. Moore MG, Kearsly G. Distance Education: A Systems View. Belmont: Wadsworth; 1995.

15. Andrade FM. O Programa de Saúde da Família no Ceará. Fortaleza: Expressão;1998.

\section{CONTRIBUIÇÃO DOS AUTORES}

Henk T. Van Der Molen foi responsável pela concepção do estudo, interpretação dos dados e revisão geral do texto. José Batista Cisne Tomaz foi responsável pela revisão da literatura, coleta e análise dos dados e redação do texto.

\section{CONFLITO DE INTERESSES}

Declarou não haver.

\section{ENDEREÇO PARA CORRESPONDÊNCIA}

José Batista Cisne Tomaz

Av. Beira-Mar, 4344 — apto 1102

Mucuripe - Fortaleza

CEP 60165-121 - CE

E-mail: batistatomaz@gmail.com 